\title{
Escuela en casa: Motivos y resultados
}

\section{Home school: Reasons and results}

Andrés Chávez

Universidad Internacional del Ecuador, Ecuador

Daniel Jurado

Universidad Internacional del Ecuador, Ecuador

Autor por Correspondencia: anchavezer@uide.edu.ec

Fecha de recepción: 4 de Marzo de 2017 - Fecha de aceptación: 5 de Junio de 2017

\section{Resumen}

En el artículo vamos a responder tres preguntas 1) ¿Quién y por qué deciden educar a los hijos en casa?, 2) ¿Cuáles son los efectos sociales que tienen al estudiar en casa? Y 3) ¿Cuáles son los resultados académicos que obtienen? En el texto se hablará de los homeschoolers y nos referimos así a los niños que por decisión de sus padres estudian en casa en vez de ir a una escuela.

Palabras claves: escuela; casa; educación; homeschool; enseñanza; socializar

\begin{abstract}
In the article, we are going to answer three questions: 1) who and why do they choose to educate their children at home? 2) What are the social effects they have when studying at home? And 3) What are the academic results they get? In the text, will be speak of the homeschoolers and we refer to the children that by their parents' decision they study at home instead of going to a school.
\end{abstract}

Key words: school; home; education; homeschool; teaching; socialize 


\section{Introducción}

En el resto del mundo, al igual que en Ecuador, existe una metodología de enseñanza a los niños que es "homeschool" o escuela en casa. Si bien es legal en el país, no es una metodología muy usada o bien vista localmente. Sin embargo, en otros países, como Estados Unidos, es una metodología usada por 1.5 millones de familias aproximadamente (Ray, 2009). Esta forma de estudio puede ser cuestionada de varias maneras, como: ¿quién decide estudiar o educar en casa?, ¿por qué toman este camino en vez de enviarlo a una escuela pública o privada?, ¿Cómo les afecta esta forma de estudio en su vida social?, ¿los padres están realmente preparados para enseñarles a sus hijos lo necesario?, ¿Cuáles son los resultados académicos cuando los jóvenes entran a las universidades?, y más dudas que alguien puede tener. Debido al cuestionamiento que existe en la actualidad, muchos estudios se han desarrollado a través de este tema, dando mejores resultados de los que uno esperaría. El siguiente artículo se va a dividir en tres partes: 1) Quién y Por qué, 2) Entorno Social y 3) Resultados académicos.

\section{Quién y Por qué}

Una de las preguntas que más se realizan cuando se habla de la escuela en casa es quién toma la decisión, puede ser el niño, el padre. Y cuando toman esta decisión por qué la toman, se debe a una tradición familiar, una creencia del tomador de la decisión, el entorno, la situación financiera.

Según un estudio realizado en Estados Unidos por Deborah Grubb (1998) a varias familias que crían a sus hijos bajo esta metodología de enseñanza, el 98\% de las familias creen que de esta manera se puede obtener mejores resultados de los que se obtiene en las escuelas. También resalta que una de las razones se debe a creencias en el desarrollo moral de los jóvenes, como la moral o valores religiosos:

"homeschooling brings families closer together, that home-schooling provides students with "proper" social, moral, and religious values, and that there is an advantage of having a flexible schedule to teach the desired curriculum" (2001)

Revisando esto, vemos que la mayoría de las razones por las cuales los padres deciden enseñar la escuela en casa se debe por creencias en un mejor desarrollo, tanto académico como moral. En el estudio se realizó una pregunta que era "¿Qué tiene que cambiar las escuelas públicas para atraer nuevamente a los jóvenes?" (Grubb, 1998). La mayoría de padres encuestados indicaron que las escuelas deben de tener principios religiosos para todo lo que concierne a las operaciones de las escuelas. También, indicaron que en las escuelas no enseñan disciplina y los jóvenes tienen poder sobre los profesores y administradores.

A pesar de que los padres toman esta decisión por beneficios que este tipo de enseñanza les da a los jóvenes, también encuentran desventajas en el sistema. Unas de las más repetidas son sobre la parte financiera, en cómo los padres pagan impuestos para la educación y no pueden recibir el beneficio que ofrece el gobierno, ya que toman la decisión que la educación sea en casa y no en las escuelas públicas. Vale indicar que en Estados Unidos las escuelas públicas son las principales recursos de educación que las personas acceden. Otra desventaja que encuentran, es 
que los padres que se dedican a enseñarles a sus hijos en casa no tienen tiempo libre, debido al tiempo que usan para esta actividad. Por último, una de las mayores preocupaciones es la falta de relación que tienen los jóvenes con sus "peers" (pares o iguales). Debido a esto, es que buscan involucrar a los hijos en actividades fuera de casa como: un deporte, actividades de la iglesia, cursos, u otras opciones.

\section{Entorno Social}

El desarrollo social que tendrá el joven que estudia en casa, es otra de las preocupaciones que tienen las partes de intereses, como el gobierno y la sociedad. Esta inquietud salta debido a que los niños no se relacionan con sus iguales o "peer" en una etapa de desarrollo. Como se mencionaba en la Primera Parte del texto, la poca socialización que tengan los jóvenes dentro de la casa es una de las preocupaciones o debilidades que los padres pueden encontrar en la modalidad.

Debido a que la socialización es mínima, los padres buscan integrar a los niños dentro de actividades extracurriculares. Aunque las actividades extracurriculares pueden ser buena para el desarrollo de los jóvenes, esta no es tan positiva cuando se trata de "homeschoolers" en actividades extracurriculares de las escuelas públicas para los jóvenes, sus familias, los alumnos de las escuelas, sus familias y la administración (Dawn, 2012, pág. 1). También, al momento de ir a seleccionar una actividad extracurricular a las escuelas, solo se les permite los deportes, más no las demás como arte, laboratorio, idiomas, etcétera. Por esto último, los padres buscan actividades fueras de las escuelas como los scouts, o en las iglesias.

Al momento en que los jóvenes deciden escoger un extracurricular de una escuela pública, hay que pensar en que tan bien se van a adaptar a los demás niños de su edad. También, hay que indicar o enseñar a los niños que tiene que respetar a las autoridades de estas escuelas a las cuales van a asistir (Dawn, 2012). A esto puede ser que no estén acostumbrados debido a que su educación es directa con sus padres, aunque esto no quite que tengan una buena educación.

Finalmente, vemos que hay alternativas para los padres e hijos que deciden estudiar en casa para poder socializar. Esta va a traer cambios positivos al desarrollo de cada uno, a pesar de que el encuentro con sus peers no sea día a día (Davis, 2005).

\section{Resultados Académicos}

Después de ver que los resultados sociales de los jóvenes que estudian en casa son mejores de lo esperado, puede surgir la pregunta de los resultados académicos de estos jóvenes al momento de entrar a la universidad o "college". Este es un tema delicado debido a que la enseñanza que obtienen los jóvenes en casa, en su mayoría, proviene solo de los padres y no de personas especializadas como los profesores en las escuelas.

Sin embargo, los resultados académicos de los homeschoolers son mucho mayor a los resultados de los estudias de las escuelas. Según un estudio realizado por Brian Ray, especialista en el campo, indica que los resultados promedios obtenido por los homeschoolers es de 80/100, mientras que los estudiantes de las escuelas obtienen un promedio de 50/100 (2009). 


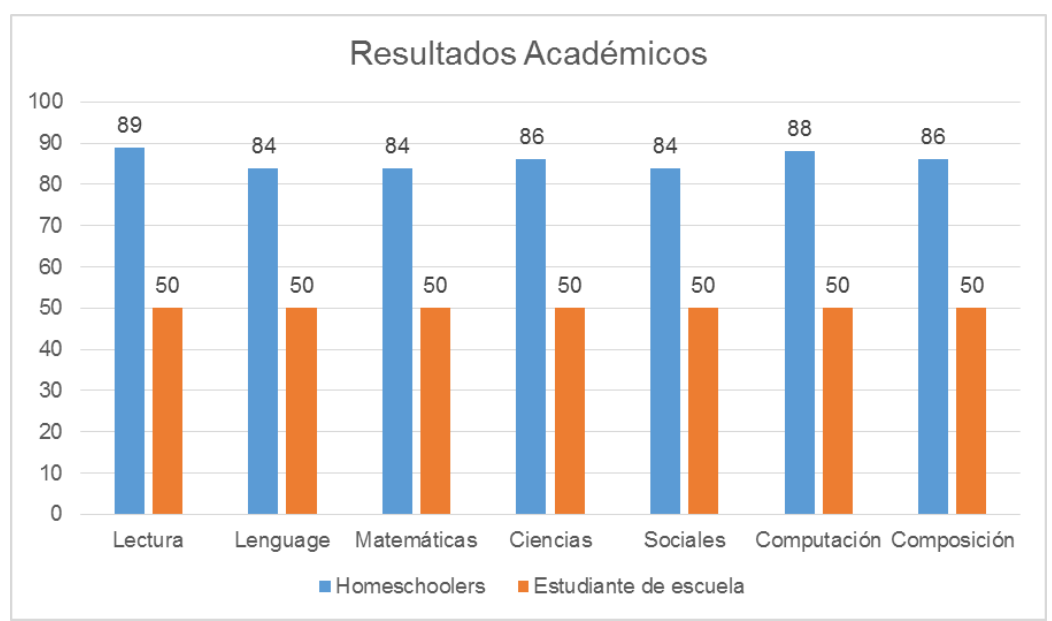

Fuente: Ray, 2009

Estos resultados se obtienen por varios factores, uno de ellos se debe a que la enseñanza en casa se encuentra regularizada al igual que las escuelas. Hay parámetros y pruebas que tienen que rendir para poder seguir avanzando y en un futuro poder entrar a una universidad. Otro factor, y uno de los más importantes, es que el joven, siendo el estudiante, tiene un mejor entorno para estudiar. En esta modalidad de enseñanza "la escuela" se adapta a las capacidades y metodologías que al estudiante se le hace más fácil entender, mientras que en las escuelas el estudiante debe de adaptarse al ritmo y metodología que tiene cada profesor.

\section{Conclusión}

En conclusión, vemos que esta metodología de enseñanza, que no es nueva en el mundo, tiene sus pros y contras. Pudiendo resaltar lo positivo sobre lo negativo, como los resultados académicos, siendo estos mejores que los estudiantes de escuelas. También, vemos como los resultados de socialización tampoco son negativos, gracias a las diferentes actividades extracurriculares que pueden ingresar los jóvenes a realizar. Finalmente, para que esta metodología de enseñanza sea utilizada y bien vista en Ecuador, primero tendrá que ser bien regularizada desde las entidades que le competen, para de esta manera, tener resultados parecidos a los países que ya la aplican.

\section{Bibliografía}

(Noviembre de 2001). The Social Development Of The Home Educated Learner In The Primary School Phase. Sur Africa.

(Noviembre de 2012 ). Homeschool Participation In Public School Extracurricular . Lynchburg.

Apostoleris, N. H. (00 de 04 de 1999). The Development of Children's Motivation in the Homeschool Setting. Alburquerque, Estados Unidos.

Bergstrom, L. (Mayo de 2012). What Effect Does Homeschooling Have on the Social Development and Test Scores of Students? Winsconsi, Estados Unidos. 
Gomez, S. (s.f.). Homework in Learning Process.

Gray, P., \& Riley, G. (Enero de 2013). The Challenges and Benefits of Unschooling, According to 232 Families Who Have Chosen that Route. Boston, Estados Unidos .

Grubb, D. (00 de 11 de 1998). Homeschooling: Who and Why? New Orleans, Los Angeles, Estados Unidos.

Heidemann, L. (08 de Febrero de 2008). Lesson Design and Biblical Reasoning for Homeschool Parents. Colorado, Estados Unidos.

Huerta, L. A., \& Gonzales, M. F. (04 de Enero de 2008). Cyber and HomeSchool Charter Schools: How States are Defining New Forms of Public Schooling. Estados Unidos.

Lundy, G. (Marzo de 2014). I'm Keeping My Son Home”: African American Males and the Motivation to Homeschool. Estados Unidos: Journal of African American.

Magno, C. (Mayo de 2012). Psychological Theories, Practices, and Ideas in the Educational Context . Philippines.

Mathis, A. B. (07 de Octubre de 2016). School Choice: Why Parents Choose Charter, Private, and Homeschool Options. Georgia, Estados Unidos: Georgia Southern University.

Meier, C., \& Lemmer, E. (Mayo de 2015). What do parents really want? Parents' perceptions of their children's schooling. Petroria, Sur Africa: South African Journal of Education.

Okeke, C. I. (Agosto de 2014). Effective home-school partnership: Some strategies to help strengthen parental involment. Sur Africa: South African Journal of Education.

Ray, B. D. (2004). Homeschoolers on to College: . Salem, Oregon, Estados Unidos.

Ray, B. D. (2009). Homeschool Progress Report 2009: Academic Achievment and Demographics. Salem, Oregon, Estados Unidos: National Home Education Research Institute.

Ray, B. D. (03 de Febrero de 2010). Academic Achievement and Demographic Traits of Homeschool Students: A National Study. Estados Unidos.

Ray, B. D. (06 de Enero de 2015). Ph D. Research Facts On Homeschooling. Salem , Oregon, Estados Unidos: National Home Education Research Institute.

Smith, J. (s.f.). Homeschool's Upper Hand over Public School. 